\title{
HIGHLIGHTS
}

PREVENTION

\section{Fibrates reduce risk of coronary events}

Randomized controlled trials of fibrate therapy have yielded variable results. Although these agonists of peroxisome proliferator receptor alpha (PPAR- $\alpha$ ) are known to reduce triglyceride levels and increase HDL-cholesterol concentration, they have not been consistently associated with reduced vascular risk. Furthermore, some studies have raised concerns about fibrate toxicity. A new meta-analysis of fibrate trials by Vlado Perkovic and co-workers is, therefore, a welcome addition to the literature. "We observed the results of the FIELD and ACCORD studies with great interest," explains Dr Perkovic, "[and] we were interested to see how these results compared with earlier studies of fibrates." The investigators showed that fibrates were associated with a reduced risk of cardiovascular (predominantly coronary) events in patients with a broad range of baseline characteristics.
The meta-analysis included

18 published randomized controlled trials, which enrolled a total of 45,058 patients (individual sample sizes ranged between 81 and 10,627). The earliest trial was published in 1971, and the most recent in 2010. Several different fibrates were studied-clofibrate was used in seven trials, bezafibrate in four, fenofibrate and gemfibrozil were each assessed in three trials, and etofibrate in one. Some heterogeneity also existed in the inclusion criteria for the studies: six studies only enrolled patients with diabetes, six had all-male cohorts, and eight required that patients had specific lipid profiles.

Fibrate therapy was associated with $13 \%$ and $10 \%$ reductions in the risk of coronary events (composite of myocardial infarction and coronary mortality) and cardiovascular events (composite of myocardial infarction and stroke), respectively, when compared with placebo. However, fibrate therapy did not

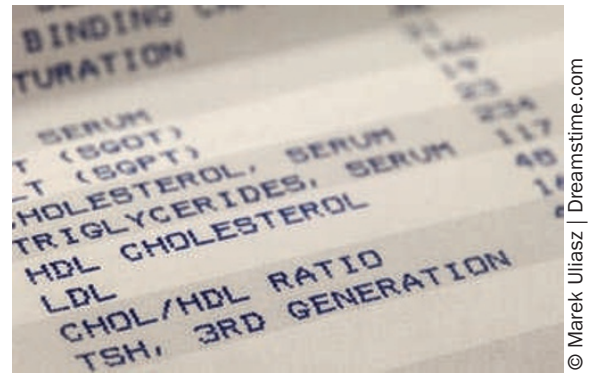

seem to reduce the incidence of all-cause mortality, vascular mortality, sudden death, coronary revascularization, or stroke. No significant increase in fibraterelated adverse events was identified. "The effects [of fibrate therapy] correlated well with the magnitude of triglyceride lowering," comments Dr Perkovic, "and appeared greater in people with high triglyceride levels, suggesting that these people may obtain even larger benefits."

\section{Alexandra King}

Original article Jun, M. et al. Effects of fibrates on cardiovascular outcomes: a systematic review and metaanalysis. Lancet 375, 1875-1884 (2010) 